\title{
Pd/C-Catalyzed Oxidative Alkylation of Secondary Alcohols with Primary Alcohols
}

\author{
Chan Sik Cho, ${ }^{\dagger, *}$ Wen Xiu Ren, and Sang Chul Shim* \\ ${ }^{\dagger}$ Research Institute of Industrial Technology, Kyungpook National University, Daegu 702-701, Korea. ${ }^{*}$ E-mail: cscho@knu.ac.kr \\ Department of Applied Chemistry, College of Engineering, Kyungpook National University, Daegu 702-701, Korea \\ EE-mail:scshim@knu.ac.kr \\ Received August 11, 2005
}

Key Words : Aldol reaction, Oxidative alkylation, Pd/C catalyst, Primary alcohols, Secondary alcohols

Transition metal-catalyzed carbon-carbon coupling protocol has been widely used as a powerful tool in synthetic organic chemistry. ${ }^{1}$ In connection with this report, during the course of our studies directed towards a ruthenium-catalyzed carbon-carbon bond forming reaction, ${ }^{2-5}$ we recently found a new ruthenium-catalyzed coupling reactions between ketones (or secondary alcohols) and primary alcohols. The coupling of ketones A with primary alcohols B preferentially afforded coupled ketones $\mathbf{C}$ (Scheme 1, route a) $)^{2,6,7}$ or coupled secondary alcohols $\mathbf{D}$ (Scheme 1 , route $b)^{3}$ according to the molar ratio of $\mathbf{B}$ to $\mathbf{A}$. In addition, secondary alcohols $\mathbf{E}$ were also found to be coupled with $\mathbf{B}$ to afford $\mathbf{D}$ (Scheme 1 , route $\mathbf{c}){ }^{4}$ Under these circumstances, ${ }^{8}$ this report describes a new $\mathrm{Pd} / \mathrm{C}$-catalyzed coupling route between $\mathbf{E}$ and $\mathbf{B}$ leading to oxidative coupled products $\mathbf{C}$ (Scheme 1, route d). ${ }^{9}$

The results of several attempted oxidative alkylation of 1phenylethanol (1a) with butanol (2a) under several conditions are listed in Table 1 . Treatment of $\mathbf{1 a}$ with 2 equiv. of $2 \mathbf{a}$ in the presence of a catalytic amount of $5 \% \mathrm{Pd} /$ $\mathrm{C}(5 \mathrm{~mol} \%)$ along with $\mathrm{KOH}$ in dioxane for $40 \mathrm{~h}$ afforded oxidative coupled product 1-phenylhexan-1-one (3a), coupled secondary alcohol 1-phenylhexan-1-ol (4), and acetophenone (5) in $2 \%, 3 \%$, and $2 \%$ yields, respectively, with low conversion of 1a (run 1). However, when 1-decene was further added as a sacrificial hydrogen acceptor, the reaction rate was enhanced toward 3a and $\mathbf{5}$ formation with complete conversion of $\mathbf{1 a}$, whereas $\mathbf{4}$ remains nearly constant (run 2). It appears that 1-decene works as a sacrificial hydrogen acceptor for a faster regeneration of $[\mathrm{Pd}]$ from $[\mathrm{Pd}] \mathrm{H}_{2}$ generated during the course of the initial oxidations of $\mathbf{1 a}$ to $\mathbf{5}$ and $\mathbf{2 a}$ to butyraldehyde. ${ }^{10}$ The molar

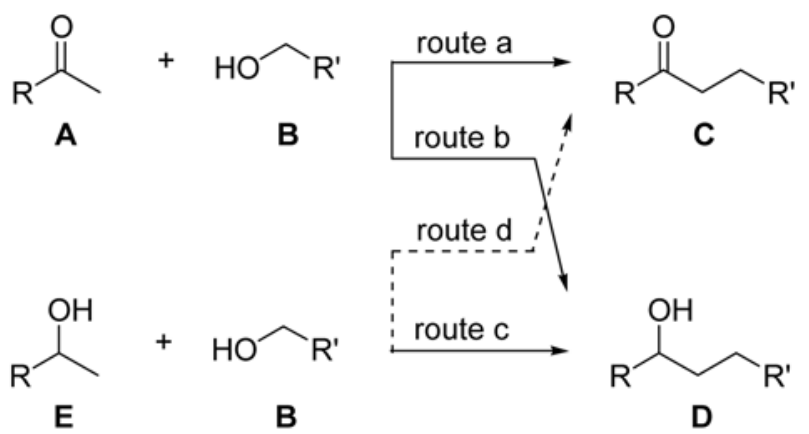

Scheme 1 ratio of $\mathbf{2 a}$ to $1 \mathbf{a}$ affects the product distribution (runs 3,4 ) The oxidized product $\mathbf{5}$ was formed in preference to $\mathbf{3 a}$ and $\mathbf{4}$ under lower molar ratio $([\mathbf{2 a}] /[\mathbf{1} \mathbf{a}]=1)$ (run 3) and a lower selectivity between coupled products was observed with higher molar ratio $([\mathbf{2 a}] /[\mathbf{1} \mathbf{a}]=3)$ (run 4). In spite of further elaboration for the optimization of reaction conditions (run $5,6)$, the best result in terms of the yield of oxidative alkylated product $\mathbf{3 a}$ and the selectivity of $\mathbf{3 a}$ to $\mathbf{4}$ is best accomplished under the standard set of condition shown in run 2 of Table 1.

Given the controlled reaction conditions, various secondary alcohols $\mathbf{1}$ and primary alcohols $\mathbf{2}$ were employed to investigate the reaction scope. The representative results are summarized in Table 2 . The reactions of 1 a with various straight and branched primary alcohols 2a-i gave the corresponding oxidative alkylated ketones 3a-i in the range of $43-83 \%$ yields with the concomitant formation of a considerable amount of acetophenone on GLC analysis. The reaction yield and rate were increased with the increase of the straight alkyl chain length in $\mathbf{2}$. The reaction proceeds likewise with aryl(methyl) carbinols $\mathbf{1 b}$-d to afford the corresponding coupled ketones $\mathbf{3} \mathbf{j}-\mathbf{l}$. On the other hand, with cyclic carbinol 1-tetralol (1e), which has methylene reaction

Table 1. Optimization of conditions for the reaction of $\mathbf{1 a}$ with $\mathbf{2} \mathbf{a}^{a}$
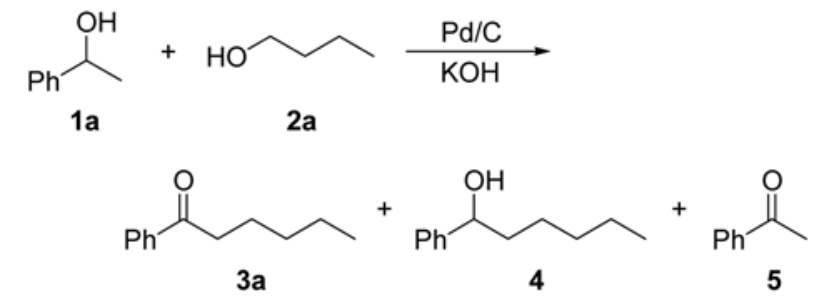

\begin{tabular}{ccccccc}
\hline \multirow{2}{*}{ Run } & & \multirow{2}{*}{$\mathbf{2 a}] /[\mathbf{1 a}]$} & Additive & $\begin{array}{c}\text { Conv. }(\%)^{b} \\
\text { of 1a }\end{array}$ & \multicolumn{3}{c}{ Yield (\%) } \\
\cline { 5 - 7 } & & & & $\mathbf{3 a}$ & $\mathbf{4}$ & $\mathbf{5}^{b}$ \\
\hline 1 & 2 & - & 21 & 2 & 3 & 3 \\
2 & 2 & 1 1-decene & 99 & $60-62^{c}$ & $2-4^{c}$ & $26-32^{c}$ \\
3 & 1 & $1-$ decene & 100 & 23 & 1 & 56 \\
4 & 3 & 1 1-decene & 95 & 41 & 19 & 23 \\
$5^{d}$ & 2 & 1-decene & 95 & 29 & 5 & 50 \\
$6^{e}$ & 2 & 1-decene & 98 & 47 & 3 & 24 \\
\hline
\end{tabular}

${ }^{a}$ Reaction conditions: 1a $(1 \mathrm{mmol}), 5 \% \mathrm{Pd} / \mathrm{C}(0.05 \mathrm{mmol}), \mathrm{KOH}(3$ $\mathrm{mmol})$, 1-decene $(5 \mathrm{mmol})$, dioxane $(2 \mathrm{~mL}), 100{ }^{\circ} \mathrm{C}$, for $40 \mathrm{~h}$. ${ }^{b}$ Determined by GLC. ${ }^{c}$ Several runs. ${ }^{d}$ For $24 \mathrm{~h} .{ }^{e} \mathrm{Pd} / \mathrm{C}(0.02 \mathrm{mmol})$. 
Table 2. Pd/C-catalyzed oxidative alkylation of $\mathbf{1}$ with $\mathbf{2}^{a}$

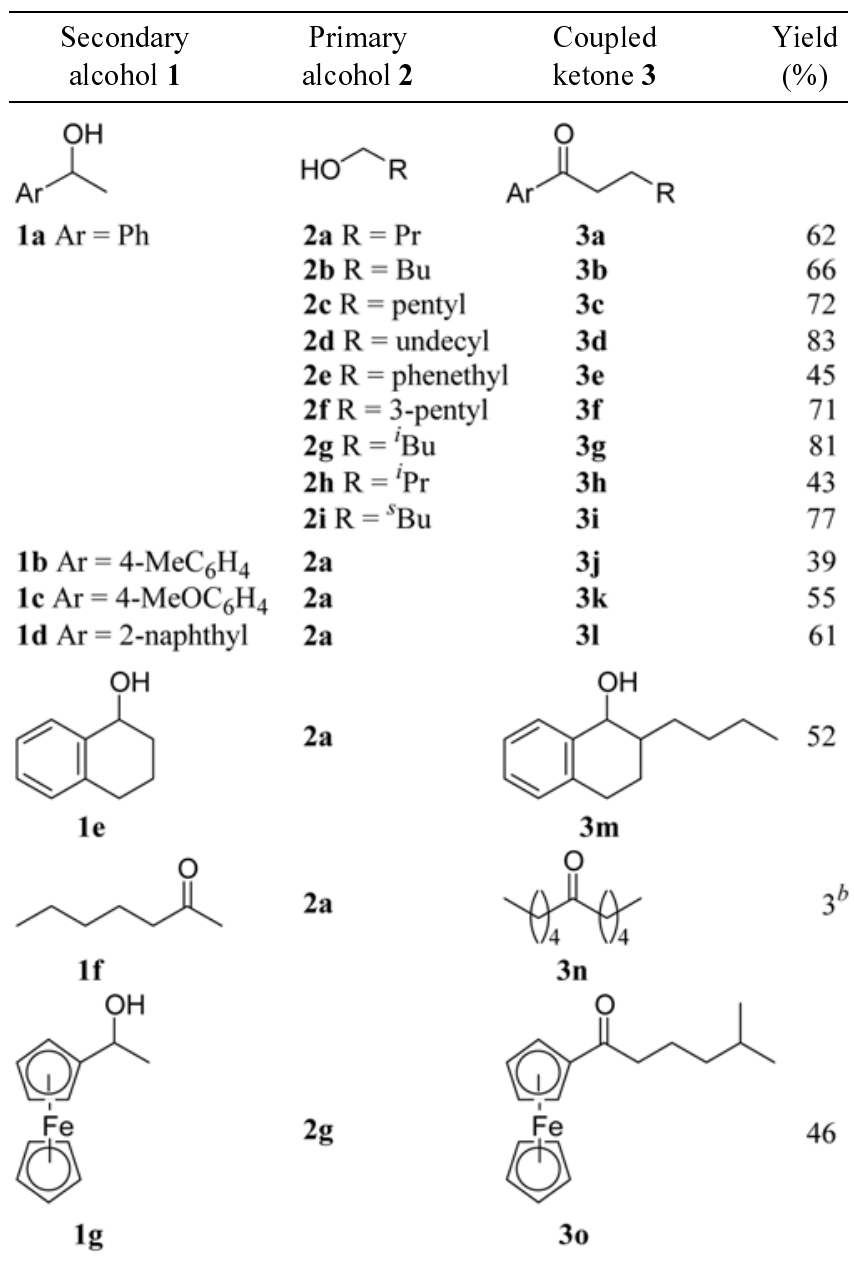

${ }^{a}$ Reaction conditions: $1(1 \mathrm{mmol}), 2(2 \mathrm{mmol}), 5 \% \mathrm{Pd} / \mathrm{C}(0.05 \mathrm{mmol})$, $\mathrm{KOH}(3 \mathrm{mmol}), 1$-decene $(5 \mathrm{mmol})$, dioxane $(2 \mathrm{~mL}), 100{ }^{\circ} \mathrm{C}$, for $40 \mathrm{~h}$. ${ }^{b} \mathrm{GLC}$ yield.

site, coupled carbinol 2-butyl-1-tetralol was absolutely produced in preference to usual coupled ketone. This result indicates that the carbonyl reduction of benzo-fused cyclic ketone is faster than that of acyclic ketone under the present $\mathrm{Pd} / \mathrm{C}$-catalyzed conditions. From alkyl(methyl) carbinol 1f, neither coupled ketone nor coupled carbinol were satisfactorily formed. In the reaction of 1-ferrocenylethanol (1g), the oxidative alkylation occurred under the employed conditions to give coupled ketone along with acetylferrocene (46\% yield).

In summary, it has been shown that aryl(methyl) carbinols undergo an oxidative coupling with primary alcohols in the presence of a catalytic amount of $\mathrm{Pd} / \mathrm{C}$ along with $\mathrm{KOH}$ to give the corresponding coupled ketones in moderate to good yields. The present reaction is a straightforward one-pot process for the oxidative alkylation of secondary alcohols. ${ }^{11}$

\section{Experimental Section}

${ }^{1} \mathrm{H}$ and ${ }^{13} \mathrm{C}$ NMR (400 and $100 \mathrm{MHz}$ ) spectra were recorded on a Bruker Avance Digital 400 spectrometer using TMS as an internal standard. GLC analyses were carried out with a Shimadzu GC-17A instrument equipped with a CBP10-S25-050 column (Shimadzu, fused silica capillary column, $0.33 \mathrm{~mm} \times 25 \mathrm{~m}, 0.25 \mu \mathrm{m}$ film thickness) using nitrogen as carrier gas. The isolation of pure products was carried out via thin layer chromatography (silica gel 60 $\mathrm{GF}_{254}$, Merck). Commercially available organic and inorganic compounds were used without further purification.

General experimental procedure. A mixture of $\mathbf{1 a}$ (0.122 g, $1 \mathrm{mmol}), 2 \mathrm{a}(0.148 \mathrm{~g}, 2 \mathrm{mmol})$, palladium, $5 \mathrm{wt} . \%$ on activated carbon $(0.106 \mathrm{~g}, 0.05 \mathrm{mmol}), \mathrm{KOH}(0.168 \mathrm{~g}, 3$ $\mathrm{mmol})$ and 1-decene $(0.701 \mathrm{~g}, 5 \mathrm{mmol})$ in dioxane $(2 \mathrm{~mL})$ was placed in a $5 \mathrm{~mL}$ screw-capped vial and allowed to react at $100{ }^{\circ} \mathrm{C}$ for $40 \mathrm{~h}$. The reaction mixture was filtered through a short silica gel column (ethyl acetate-hexane mixture) to eliminate inorganic salts. To the extract was added appropriate amount of undecane as an internal standard and analyzed by GLC for the determination of the conversion of 1a $(99 \%)$ and the yield of $5(26 \%)$. Removal of the solvent left a crude mixture, which was separated by thin layer chromatography ( silica gel, ethyl acetate-hexane mixture) to give 3a $(0.109 \mathrm{~g}, 62 \%)$ and $4(0.007 \mathrm{~g}, 4 \%)$. All products prepared by the above procedure were identified by comparison with samples noted in our recent report except for $\mathbf{3 b}, \mathbf{3 d}, \mathbf{3 f}, \mathbf{3} \mathbf{j}-\mathbf{l}$, and $\mathbf{3 o}$. $^{2,5,6}$

1-Phenylheptan-1-one (3b). ${ }^{12}$ Oil; ${ }^{1} \mathrm{H}$ NMR $\left(\mathrm{CDCl}_{3}\right) \delta$ $0.89(\mathrm{t}, J=6.5 \mathrm{~Hz}, 3 \mathrm{H}), 1.31-1.40(\mathrm{~m}, 6 \mathrm{H}), 1.69-1.77(\mathrm{~m}$, 2H), 2.95 (t, $J=7.5 \mathrm{~Hz}, 2 \mathrm{H}), 7.43-7.46(\mathrm{~m}, 2 \mathrm{H}), 7.54(\mathrm{t}, J=$ $7.5 \mathrm{~Hz}, 1 \mathrm{H}), 7.95(\mathrm{~d}, J=7.6 \mathrm{~Hz}, 2 \mathrm{H}) ;{ }^{13} \mathrm{C} \mathrm{NMR}\left(\mathrm{CDCl}_{3}\right) \delta$ 14.4, 22.9, 24.7, 29.4, 32.1, 39.0, 128.4, 128.9, 133.2, 137.5, 200.9.

1-Phenyltetradecan-1-one (3d). Solid (hexane); mp 53$53.5{ }^{\circ} \mathrm{C}$ (lit. $\left.{ }^{13} 52.5{ }^{\circ} \mathrm{C}\right) ;{ }^{1} \mathrm{H}$ NMR $\left(\mathrm{CDCl}_{3}\right) \delta 0.88(\mathrm{t}, J=6.8$ $\mathrm{Hz}, 3 \mathrm{H}), 1.26-1.40$ (m, 20H), 1.70-1.77 (m, 2H), 2.96 (t, $J=$ $7.5 \mathrm{~Hz}, 2 \mathrm{H}), 7.43-7.47$ (m, 2H), 7.52-7.57 (m, 1H), 7.94$7.97(\mathrm{~m}, 2 \mathrm{H}) ;{ }^{13} \mathrm{C} \mathrm{NMR}\left(\mathrm{CDCl}_{3}\right) \delta 14.50,23.08,24.78$, 29.74, 29.77, 29.88, 29.90, 30.01, $30.03(\times 2), 30.06,32.31$, 39.02, 128.44, 128.91, 133.21, 137.51, 200.97.

4-Ethyl-1-phenylhexan-1-one (3f). ${ }^{14}$ Oil; ${ }^{1} \mathrm{H} \quad \mathrm{NMR}$ $\left(\mathrm{CDCl}_{3}\right) \delta 0.88(\mathrm{t}, J=7.3 \mathrm{~Hz}, 6 \mathrm{H}), 1.26-1.38(\mathrm{~m}, 5 \mathrm{H}), 1.67-$ $1.72(\mathrm{~m}, 2 \mathrm{H}), 2.94(\mathrm{t}, J=7.8 \mathrm{~Hz}, 2 \mathrm{H}), 7.43-7.47(\mathrm{~m}, 2 \mathrm{H})$, $7.54(\mathrm{t}, J=7.5 \mathrm{~Hz}, 1 \mathrm{H}), 7.95-7.97(\mathrm{~m}, 2 \mathrm{H}) ;{ }^{13} \mathrm{C} \mathrm{NMR}$ $\left(\mathrm{CDCl}_{3}\right) \delta 11.2,25.7,27.6,36.5,40.5,128.4,128.9,133.2$, 137.5, 201.3.

1-(4-Methylphenyl)hexan-1-one (3j). ${ }^{15}$ Oil; ${ }^{1} \mathrm{H}$ NMR $\left(\mathrm{CDCl}_{3}\right) \delta 0.91(\mathrm{t}, J=7.0 \mathrm{~Hz}, 3 \mathrm{H}), 1.34-1.38(\mathrm{~m}, 4 \mathrm{H}), 1.69-$ $1.77(\mathrm{~m}, 2 \mathrm{H}), 2.40(\mathrm{~s}, 3 \mathrm{H}), 2.93(\mathrm{t}, J=7.5 \mathrm{~Hz}, 2 \mathrm{H}), 7.25(\mathrm{~d}, J$ $=8.0 \mathrm{~Hz}, 2 \mathrm{H}), 7.86(\mathrm{~d}, J=8.0 \mathrm{~Hz}, 2 \mathrm{H}) ;{ }^{13} \mathrm{C} \mathrm{NMR}\left(\mathrm{CDCl}_{3}\right) \delta$ 14.3, 22.0, 22.9, 24.6, 32.0, 38.9, 128.6, 129.6, 135.0, 143.9, 200.7.

1-(4-Methoxyphenyl)hexan-1-one (3k). ${ }^{15}$ Solid (hexane); mp 32-35 ${ }^{\circ} \mathrm{C}$; ${ }^{1} \mathrm{H}$ NMR $\left(\mathrm{CDCl}_{3}\right) \delta 0.91(\mathrm{t}, J=7.0 \mathrm{~Hz}, 3 \mathrm{H})$, 1.34-1.38 (m, 4H), 1.69-1.76 (m, 2H), $2.90(\mathrm{t}, J=7.5 \mathrm{~Hz}$ 2H), 3.87 (s, 3H), 6.93 (d, $J=8.8 \mathrm{~Hz}, 2 \mathrm{H}), 7.94(\mathrm{~d}, J=8.8$ $\mathrm{Hz}, 2 \mathrm{H}) ;{ }^{13} \mathrm{C} \mathrm{NMR}\left(\mathrm{CDCl}_{3}\right) \delta 14.0,22.6,24.3,31.6,38.3$, $55.4,113.7,130.2,130.3,163.3,199.3$.

1-(2-Naphthalenyl)hexan-1-one (3I). ${ }^{16}$ Solid (hexane); mp 67-68 ${ }^{\circ} \mathrm{C}$; ${ }^{1} \mathrm{H} \mathrm{NMR}\left(\mathrm{CDCl}_{3}\right) \delta 0.93(\mathrm{t}, J=7.0 \mathrm{~Hz}, 3 \mathrm{H})$, 
1.37-1.42 (m, 4H), 1.76-1.84 (m, 2H), $3.09(\mathrm{t}, J=7.5 \mathrm{~Hz}$, $2 \mathrm{H}), 7.52-7.60(\mathrm{~m}, 2 \mathrm{H}), 7.88(\mathrm{t}, J=7.8 \mathrm{~Hz}, 2 \mathrm{H}), 7.96(\mathrm{~d}, J=$ $8.0 \mathrm{~Hz}, 1 \mathrm{H}), 8.03$ (dd, $J=8.5$ and $1.5 \mathrm{~Hz}, 1 \mathrm{H}), 8.46(\mathrm{~s}, 1 \mathrm{H})$; ${ }^{13} \mathrm{C} \mathrm{NMR}\left(\mathrm{CDCl}_{3}\right) \delta 14.4,23.0,24.6,32.0,39.1,124.4$, $127.1,128.1,128.7,128.8,129.9,130.0,133.0,134.8$, 135.9, 201.0.

1-Ferrocenyl-5-methylhexan-1-one (3o). Brown oil; ${ }^{1} \mathrm{H}$ $\operatorname{NMR}\left(\mathrm{CDCl}_{3}\right) \delta 0.91(\mathrm{~d}, J=6.5 \mathrm{~Hz}, 6 \mathrm{H}), 1.23-1.29(\mathrm{~m}, 2 \mathrm{H})$, 1.55-1.63 (m, 1H), 1.67-1.75 (m, 2H), $2.68(\mathrm{t}, J=7.5 \mathrm{~Hz}$, 2H), 4.19 (s, 5H), 4.48 (t, $J=2.0 \mathrm{~Hz}, 2 \mathrm{H}), 4.78$ (t, $J=2.0$ $\mathrm{Hz}, 2 \mathrm{H}) ;{ }^{13} \mathrm{C} \mathrm{NMR}\left(\mathrm{CDCl}_{3}\right) \delta 22.89,22.95,28.35,39.20$, 40.37, 69.71, 70.11, 72.48, 79.58, 205.14. Anal. Calcd for $\mathrm{C}_{17} \mathrm{H}_{22} \mathrm{FeO}$ : C, 68.47; H, 7.44. Found: C, 68.11; H, 7.63.

Acknowledgment. The present work was supported by BK-21 in 2003 and a Research Foundation Grant (KRF2002-070-C00055). C.S.C. gratefully acknowledges a Research Professor Grant of Kyungpook National University (2004).

\section{References}

1. (a) Metal-catalyzed Cross-coupling Reactions; Stang, P. J., Diederich, F., Eds.; Wiley-VCH: Weinheim, 1998.

2. Cho, C. S.; Kim, B. T.; Kim, T.-J.; Shim, S. C. Tetrahedron Lett. 2002, 43, 7987

3. Cho, C. S.; Kim, B. T.; Kim, T.-J.; Shim, S. C. J. Org. Chem. 2001, 66, 9022

4. Cho, C. S.; Kim, B. T.; Kim, H.-S.; Kim, T.-J.; Shim, S. C. Organometallics 2003, 22, 3608.

5. Cho, C. S.; Kim, B. T.; Lee, M. J.; Kim, T.-J.; Shim, S. C. Angew. Chem., Int. Ed. 2001, 40, 958.

6. For a $\mathrm{Pd} / \mathrm{C}$-catalyzed version for $\alpha$-alkylation of ketones by primary alcohols: Cho, C. S. J. Mol. Cat. A: Chem. 2005, 240, 55.

7. For an iridium-catalyzed version for $\alpha$-alkylation of ketones by primary alcohols: Taguchi, K.; Nakagawa, H.; Hirabayashi, T.; Sakaguchi, S.; Ishii, Y. J. Am. Chem. Soc. 2004, 126, 72

8. These methodologies could be successfully applied to modified Friedläender quinoline synthesis by the ruthenium- and iridiumcatalyzed oxidative cyclization of 2-aminobenzyl alcohol with ketones and secondary alcohols: (a) Cho, C. S.; Kim, B. T.; Kim, T.-J.; Shim, S. C. Chem. Commun. 2001, 2576. (b) Cho, C. S.; Kim, B. T.; Choi, H.-J.; Kim, T.-J.; Shim, S. C. Tetrahedron 2003, 59, 7997. (c) Motokura, K.; Mizugaki, T.; Ebitani, K.; Kaneda, K. Tetrahedron Lett. 2004, 45, 6029. (d) Taguchi, K.; Sakaguchi, S.; Ishii, Y. Tetrahedron Lett. 2005, 46, 4539.

9. For our recent report on palladium catalysis: (a) Cho, C. S.; Lim, D. K.; Heo, N. H.; Kim, T.-J.; Shim, S. C. Chem. Commun. 2004, 104. (b) Cho, C. S.; Lim, D. K.; Zhang, J. Q.; Kim, T.-J.; Shim, S. C. Tetrahedron Lett. 2004, 45, 5653.

10. For recent reviews on transition metal-catalyzed transfer hydrogenation, see: (a) Zassinovich, G.; Mestroni, G.; Gladiali, S. Chem. Rev. 1992, 92, 1051. (b) Bäckvall, J.-E.; Chowdhury, R. L.; Karlsson, U.; Wang, G. Perspectives in Coordination Chemistry; Williams, A. F.; Floriani, C.; Merbach, A. E. Eds.; VCH: New York, 1992; pp 463-486. (c) Noyori, R.; Hashiguchi, S. Acc. Chem. Res. 1997, 30, 97. (d) Naota, T.; Takaya, H.; Murahashi, S.I. Chem. Rev. 1998, 98, 2599. (e) Palmer, M.; Wills, M. Tetrahedron: Asymmetry 1999, 10, 2045.

11. The oxidative alkylation of secondary alcohol conventionally can be performed via two step-by-step unit transformations such as oxidation of secondary alcohol to ketone and an appropriate alkylation.

12. Shen, H.-C.; Su, H.-L.; Hsueh, Y.-C.; Liu, R.-S. Organometallics 2004, 23, 4332 .

13. Whitmore, F. C.; Schiessler, R. W.; Rowland, C. S.; Cosby, J. N. J. Am. Chem. Soc. 1947, 69, 235.

14. Rothstein, E.; Schofield, W. G. J. Chem. Soc. 1965, 4566.

15. Shimada, T.; Yamamoto, Y. J. Am. Chem. Soc. 2002, 124, 12670.

16. Kaneko, Y.; Sarker, A. M.; Neckers, D. C. Chem. Mater. 1999, 11, 170 